\title{
Thermal evolution of ferroelectric behavior in epitaxial $\mathrm{Hf}_{0.5} \mathrm{Zr}_{0.5} \mathrm{O}_{2}$
}

\author{
J. W. Adkins ${ }^{1,2,5}$, I. Fina ${ }^{3}$, F. Sánchez ${ }^{3}$, S. R. Bakaul $2^{2 *}$ and J. T. Abiade ${ }^{1,4,5}$ \\ ${ }^{1}$ Department of Civil and Materials Engineering, University of Illinois at Chicago, Chicago, IL 60607, USA \\ ${ }^{2}$ Materials Science Division, Argonne National Laboratory, Lemont, IL 60439, USA \\ ${ }^{3}$ Institut de Ciència de Materials de Barcelona (ICMAB-CSIC), Campus UAB, Bellaterra 08193, \\ Barcelona, Spain \\ ${ }^{4}$ Department of Mechanical and Industrial Engineering, University of Illinois at Chicago, Chicago, IL 60607, USA \\ ${ }^{5}$ Laboratory for Oxide Research and Education, 842 W. Taylor Street, Chicago, IL 60607, USA
}

[*] Corresponding author, sbakaul@anl.gov

\begin{abstract}
Herein we report a cryogenic-temperature study on the evolution of ferroelectric properties of epitaxial $\mathrm{Hf}_{0.5} \mathrm{Zr}_{0.5} \mathrm{O}_{2}$ thin films on silicon. Wake-up, endurance, and fatigue of these films are found to be intricately correlated, strongly hysteretic, and dependent on available thermal energy. Field-dependent measurements reveal a decrease in polarization with temperature, which has been determined not to be an intrinsic change of the material property, rather, a demonstration of the increase in the coercive bias of the material. Our findings suggest that a deficiency in thermal energy suppresses the mobility of defects presumed to be oxygen vacancies during wake-up and trapped injected charge during fatigue, which is responsible for polarization evolution during cycling. This permits accelerated wake-up and fatigue effects at high temperatures where thermal energy is abundant but delays these effects at cryogenic temperatures.
\end{abstract}


Since the discovery of ferroelectric in Si-doped $\mathrm{HfO}_{2}$ by Böscke et al in $2011^{1}$, researchers have endeavored to explore its origin and nuances, as well as its practical application in industries like electrocaloric energy storage ${ }^{2,3}$ and nonvolatile memory ${ }^{4,5}$. Following Si-doped $\mathrm{HfO}_{2}$, a number of other solid solutions were discovered to exhibit ferroelectricity, with dopants such as $\mathrm{Al}^{6}, \mathrm{Gd}^{7}, \mathrm{Sr}^{8}, \mathrm{Y}^{9}$, and most ubiquitously, $\mathrm{Zr}^{10}$. Of import to any practical application of $\mathrm{HfO}_{2}-$ based ferroelectrics is endurance. This quality is reflected in the evolution of the ferroelectric polarization with electric cycling, termed "wake-up" 11 and "fatigue"12-14. Wake-up is the process by which the ferroelectric polarization increases from a sub-optimal initial value ${ }^{11}$. Following wake-up, the polarization stabilizes for a variable number of electric field cycles, then begins to decrease as fatigue sets in ${ }^{12-14}$.

Wake-up and fatigue characteristics have been extensively studied in $\mathrm{HfO}_{2}$-based ferroelectrics grown via atomic layer deposition ${ }^{11,14,15}$, but these characteristics are less documented for pulsed laser deposition (PLD)-grown films. In the case of $\mathrm{HfO}_{2}$-based ferroelectrics prepared by ALD, the presence of mixed phases is expected to play a significant role in wake-up behavior. In their pristine state, polycrystalline $\mathrm{HfO}_{2}$ solid solutions often occur as a mixture of both a ferroelectric orthorhombic phase and a paraelectric monoclinic phase, the latter of which is believed to be partially responsible for the low initial polarization ${ }^{16}$. According to Grimley et al., exposing ferroelectric hafnia to enough switching cycles has the effect of facilitating a monoclinic-orthorhombic phase transition in the bulk, and a tetragonal-orthorhombic phase transition at the film-electrode interface ${ }^{17}$. Because neither the monoclinic nor tetragonal phase contributes to ferroelectricity in $\mathrm{HfO}_{2}$-based systems, their diminishment with cycling (and corresponding increase in the orthorhombic phase volume) increases polarization and "awakens" the material. In the case of some epitaxial $\mathrm{HfO}_{2}$, the evolution of polarization with cycling is less a matter of phase changes, and more a matter of charge-carrier domain wall interactions. This position has recently been taken by Chouprik et al. ${ }^{18}$, albeit in ALD-grown films with negligible monoclinic phase fraction, who pointed to the minimization of oxygen vacancy- and trappedcharge-mediated domain wall pinning as a plausible origin of wake-up. As PLD growth of oxide thin films often requires high-vacuum and high-temperature conditions, the resulting vacancy defect density can be quite different than in $\mathrm{HfO}_{2}$-based ferroelectrics prepared with $\mathrm{ALD}^{19,20}$, which will also influence phenomena mediated by oxygen vacancies. In both ALD- and PLDgrown films, accumulation of defects in the bulk of the film after extended cycling, e.g. by means of charge injection, results in repining of domains and the onset of fatigue.

The mobility of charged defects is strongly dependent upon temperature, which likely contributes to some of the temperature-sensitive endurance behavior already reported in hafniabased ferroelectrics. However, many of these reports investigate endurance at room temperature or higher, where charged defect mobility is high. There are few low-temperature investigations of wake-up and endurance in $\mathrm{HfO}_{2}$, the majority of which involve films grown via $\mathrm{ALD}^{21-24}$. Therefore, considering the noted differences anticipated between ALD- and PLD-grown $\mathrm{HfO}_{2}$ solid solutions, the role of charged defects on the wake-up and fatigue behavior of $\mathrm{HfO}_{2}$-based ferroelectrics may be further elucidated by careful study of epitaxial PLD-grown films. Herein we present a systematic study of the influence of temperatures down to $33 \mathrm{~K}$ on the ferroelectric properties and endurance behavior of $\mathrm{Hf}_{0.5} \mathrm{Zr}_{0.5} \mathrm{O}_{2}$. Polarization vs. voltage $(\mathrm{P}-\mathrm{V})$ hysteresis, 
positive-up-negative-down (PUND), and cyclic electric fatigue measurements are employed to depict the evolution of the material's properties with temperature and contextualize the behavior within the current understandings of wake-up and fatigue in $\mathrm{HfO}_{2}$-based ferroelectrics.

$\mathrm{Hf}_{0.5} \mathrm{Zr}_{0.5} \mathrm{O}_{2}$ thin films were deposited on $\mathrm{La}_{2 / 3} \mathrm{Sr}_{1 / 3} \mathrm{MnO}_{3} / \mathrm{LaNiO}_{3} / \mathrm{CeO}_{2} /$ yttria-stabilized zirconia-buffered $\mathrm{Si}(100)$ substrates via PLD using a 248-nm KrF excimer laser ${ }^{25,26}$. The 5.6-nm thick $\mathrm{HZO}$ ferroelectric layers were deposited at a repetition rate of $2 \mathrm{~Hz}, \mathrm{PO}_{2}=0.1 \mathrm{mbar}$, and $\mathrm{T}_{\mathrm{s}}$ $=800{ }^{\circ} \mathrm{C}$, then cooled under $0.2 \mathrm{mbar}^{\mathrm{O}_{2}}$. Platinum top electrodes of thickness $\mathrm{t}=20 \mathrm{~nm}$ and diameter $\mathrm{d}=20 \mu \mathrm{m}$ were deposited through a stencil mask with $\mathrm{dc}$ magnetron sputtering. Structural characterization of the thin films via x-ray diffraction is presented in Fig. 1, where the presence of only (00h)-oriented atomic planes, along with the peak at $2 \theta=30.4^{\circ}$ corresponding to (111)oriented orthorhombic HZO, indicates epitaxial film growth. Representative field-dependent polarization and capacitance hysteresis loops are presented in Fig. 2. and Fig. S1, respectively. The presence of switching current peaks and butterfly-shaped trend of capacitance change confirm the ferroelectric behavior of these films. We note that due to materials and electrodes quality variations, a maximum of $\pm 5 \%$ deviations from the measured polarization values is possible, as revealed by multiple measurements performed on several devices at same experimental condition.

All further temperature-dependent hysteresis loops were collected with an applied bias $\mathrm{V}_{\text {app }}$ $=3 \mathrm{~V}$, hysteresis period of $3 \mathrm{~ms}$, and frequency of $333 \mathrm{~Hz}$. Positive-Up-Negative-Down (PUND) data were collected by applying a 79-cycle set of two switching and non-switching positive and negative voltage pulses, incrementally increasing the maximum drive bias from $\mathrm{V}_{\text {app }}=0.25$ to $4 \mathrm{~V}$ $\left(\mathrm{E}_{\mathrm{app}}=0.45-7.14 \mathrm{MV} / \mathrm{cm}\right)$ which each cycle. The pulse width was fixed at $1 \mathrm{~ms}$ and pulse delay at $1000 \mathrm{~ms}$ for all cycles. Fatigue cycling was achieved with the use of a square-wave pulse profile with $\mathrm{V}_{\max }=3 \mathrm{~V}\left(\mathrm{E}_{\max }=5.36 \mathrm{MV} / \mathrm{cm}\right)$, pulse frequency of $5 \mathrm{kHz}$, and pulse width of $0.05 \mathrm{~ms}$; the number of cycles was controlled by varying the duration of the pulse application. Post-fatigue PUND measurements were collected with $\mathrm{V}_{\mathrm{app}}=3 \mathrm{~V}$, pulse width $=1 \mathrm{~ms}$, and pulse delay $=1000$ ms. For field-dependent fatigue cycling, the maximum applied bias was varied from $3 \mathrm{~V}$ to $6 \mathrm{~V}$ $\left(\mathrm{E}_{\mathrm{app}}=5.36-10.71 \mathrm{MV} / \mathrm{cm}\right)$, with the same waveform characteristics as temperature-dependent fatigue cycling.

The results of variable-temperature measurements are featured in Fig. 3. Near room temperature $(\mathrm{T}=280 \mathrm{~K}$ ), the pristine $\mathrm{HZO}$ (Fig. 3a) exhibits a memory window of approximately $12 \mu \mathrm{C} / \mathrm{cm}^{2}$ and coercive field $E_{c}$ of $\pm 1.36 \mathrm{~V}$. These low values are commonly reported in precycled $\mathrm{HfO}_{2}$-based ferroelectrics and are related to the pinned domains and unfavorable defect distribution present in their as-fabricated state ${ }^{18}$. Furthermore, the hysteresis loops do not show saturation, because the low thickness, $5.6 \mathrm{~nm}$, restricts the application of exceedingly high voltage for breakdown prevention. As the material is cooled from $280 \mathrm{~K}$ to $33 \mathrm{~K}$, the memory window and apparent coercive bias of the pristine material decrease (Fig. $3 \mathrm{~d}$ ). At T $=33 \mathrm{~K}, 2 P_{r}$ is $4.9 \mu \mathrm{C} / \mathrm{cm}^{2}$, $\sim 48 \%$ of $P_{r, 280 \mathrm{~K}}$. Similarly, the apparent $E_{c}$ decreases to $\pm 0.80 \mathrm{MV} / \mathrm{cm}$. This trend is unexpected because Landau-Devonshire Theory suggests that the polarization order parameter in ferroelectrics should increase as the system's temperature moves further below the order-disorder transition ${ }^{27}$. However, recent modeling efforts by Vopsaroiu et al predicts that a ferroelectric's coercive bias should also increase as temperature decreases ${ }^{28}$. For example, Wang et al. observed an 
approximate $50 \%$ increase in $\mathrm{Zr}$-doped $\mathrm{HfO}_{2}$ as the temperature decreased from $300 \mathrm{~K}$ to $100 \mathrm{~K}^{24}$, and based upon a theoretical model ${ }^{27}$, we anticipate an increase of similar magnitude for our own films. Thus, the fixed $5.36 \mathrm{MV} / \mathrm{cm}$ field we employ for hysteresis measurements may be insufficient to keep up with evolving coercive bias at decreasing temperature, which prevents the complete switching of the ferroelectric and leads to the decreasing polarization at cryogenic temperatures.

An additional comparison of the behavior of the switchable polarization with decreasing temperature that fixes the ratio of the applied field to the measured coercive field $\left(\mathrm{E}_{\mathrm{app}} / \mathrm{E}_{\mathrm{c}}\right)$ at about 3.5 is included in section S2 of the Supplementary Material. The polarization still decreases from $280 \mathrm{~K}$ to $33 \mathrm{~K}$, but does so to a smaller extent, decreasing by $\sim 24 \%$ at a fixed field ratio rather than by $48 \%$ at the fixed applied field previously mentioned. We suspect the apparent coercive bias reported here for hysteresis loops collected at low temperatures represent impartial switching of the ferroelectric. This is made apparent in both the lack of saturation behavior of the pristine material, as well as the PUND switching behavior featured in Fig. $3 \mathrm{~b}$. Under a $5.36 \mathrm{MV} / \mathrm{cm}$ field generated by a $3 \mathrm{~V}$ pulse at $280 \mathrm{~K}$, the positive switchable polarization is approximately 12 $\mu \mathrm{C} / \mathrm{cm}^{2}$; to achieve a similar polarization at $33 \mathrm{~K}$ requires a $\sim 4 \mathrm{~V}$ (which generates a $7.14 \mathrm{MV} / \mathrm{cm}$ field) pulse. Zhou et al. observed a similar trend of low-temperature polarization-dependence on poling field in $\mathrm{ALD}$-grown $\mathrm{Si}$-doped $\mathrm{HfO}_{2}{ }^{23}$, suggesting that this behavior is common among hafnia-based ferroelectrics regardless of growth method.

Following the 79-cycle PUND measurements (Post-PUND) at all temperatures, the HZO exhibits an increase in memory window and coercive bias - the wake-up effect. (Fig. 3c). This results from the redistribution of oxygen vacancies during PUND cycling, which de-pin the static domains present in the pristine state and enable better switching behavior ${ }^{18,29}$. Due to the suspected participation of oxygen vacancies and the influence of temperature on their mobility ${ }^{22}$, the wakeup effect is temperature-dependent, represented in Fig. 3c by the difference in hysteresis behavior at each measurement temperature. This trend is most apparent in Fig. 3d, where comparison of coercive bias and switchable polarization evolution after PUND cycling reveals a $\sim 100 \%$ increase in memory window to $\sim 24 \mu \mathrm{C} / \mathrm{cm}^{2}$ at $280 \mathrm{~K}$ and much smaller increase in memory window $(\sim 50$ $\%)$ to $7.7 \mu \mathrm{C} / \mathrm{cm}^{2}$ at $33 \mathrm{~K}$.

To determine if similar temperature trends in ferroelectric switching behavior exist in longterm performance of $\mathrm{HZO}$, a series of fatigue measurements were conducted from $293 \mathrm{~K}$ to $33 \mathrm{~K}$. Fig. 4a presents the polarization as a function of fatigue cycles for varying temperatures. Across the temperature range $\mathrm{T}=293 \mathrm{~K}$ to $\mathrm{T}=130 \mathrm{~K}$, temperature-dependent fatigue behavior is made apparent. At T = $293 \mathrm{~K}$, fatigue begins immediately following 5000 fatigue cycles, with any wakeup effects occurring in the cycles before then; device failure occurs after $10^{6}$ cycles, and no further data were acquired. At $\mathrm{T}=220 \mathrm{~K}$, wake-up again occurs within 5000 cycles, but steady fatigue onset is delayed by about 30000 cycles. Cycling at $\mathrm{T}=160 \mathrm{~K}$ and $130 \mathrm{~K}$ produces observable wake-up until about 15000 cycles, after which polarization remains relatively stable until gradual fatigue sets in after $\sim 75000$ cycles.

To model the activation energy associated with the fatigue mechanism, a temperaturedependent fatigue model first introduced by Brennan et al. ${ }^{30}$ and later adapted by Huang et al. ${ }^{31}$ is 
considered. This model is reproduced in Eq. 1, and presents the polarization $\mathrm{P}(\mathrm{N})$ of a ferroelectric as a function of the temperature-dependent Arrhenius function R(T) in Eq. 2 and the number of fatigue cycles $N$. A reinterpretation of Eq. 1 into Eq. 3 instead presents the fatigue of the ferroelectric $f(N)$ as a function of $R(T)$ and $N$, whose slope is approximately equal to $R(T)$ :

$$
\begin{gathered}
P(N)=P_{0}[1-R(T) \log (N)] \\
R(T)=A e^{\frac{-E_{a}}{K_{B} T}} \\
f(N)=1-R(T) \log (N)
\end{gathered}
$$

Here, $f(N)=\frac{P(N)}{P_{0}}$. The magnitude of the function $\mathrm{R}(\mathrm{T})$ determines the rate of fatigue at a given temperature, and we have calculated its approximate value at T = $293 \mathrm{~K}, 250 \mathrm{~K}, 220 \mathrm{~K}, 190$ $\mathrm{K}, 160 \mathrm{~K}$, and $130 \mathrm{~K}$ using the fatigue data presented in Fig. 4a. By taking the natural logarithm of Eq. 2., a linear function whose slope is proportional to the thermal activation energy is produced:

$$
\ln [R(T)]=\ln (A)-\frac{E_{a}}{K_{b}}\left(\frac{1}{T}\right)
$$

By taking the natural logarithm of $\mathrm{R}(\mathrm{T})$ at each of the temperatures and plotting them against the inverse of temperature, we calculate and approximate thermal activation energy $E_{a}$ of the mechanism influencing fatigue in our HZO film: $23.4 \mathrm{meV}$ (Fig 4a. inset). Further details regarding these calculations may be found in section S3 of the Supplementary Material. This value, while too low to be associated with oxygen vacancy movement during fatigue ${ }^{30,31}$ is close to some reported values of the thermal activation energy associated with pinning of domain walls by injected charge carriers ${ }^{15,30}$ trapped during electric field, which suggests that they may play a role in the fatigue in our material, as well.

At lower temperatures $\left(\mathrm{T}=100 \mathrm{~K}\right.$ and $\mathrm{T}=33 \mathrm{~K}$ ), no fatigue is observed after $10^{7}$ cycles, but a minuscule improvement in polarization characteristic of wake-up appears. Fig $4 \mathrm{~b}$. presents these observations as a function of temperature. At $100 \mathrm{~K}$, total remanent polarization values increase from $6.6 \mu \mathrm{C} / \mathrm{cm}^{2}$ to $9.9 \mu \mathrm{C} / \mathrm{cm}^{2}$ over $10^{7}$ cycles, presenting a $50 \%$ increase in polarization during this wake-up regime. At $33 \mathrm{~K}$, the increase is almost half as small, with total remanent polarization increasing from $4.4 \mu \mathrm{C} / \mathrm{cm}^{2}$ to $5.3 \mu \mathrm{C} / \mathrm{cm}^{2}(\sim 25 \%$ increase). At these temperatures, a deficiency in thermal energy can suppress charge mobility and delay both wake-up and fatigue.

We finally investigate the field-dependence of the switching and endurance of $\mathrm{HZO}$ at cryogenic temperatures, a series of variable-field hysteresis and fatigue measurements were conducted. From Fig. 5a., it is shown that the initial remanent polarization and coercive field increase with increasing applied field, reaching a relative maximum at $E_{\text {app, } \max }=10.71 \mathrm{MV} / \mathrm{cm}$; above this value device breakdown occurs. This suggests that ferroelectric switching is improving, as the larger field compensates for the increased coercive bias of HZO and suppressed switching at such low temperatures, despite the lack of sharp switching and saturation in the hysteresis loops acquired. In addition to suppressed switching, the behavior of the charged defects whose pinning of domain walls may contribute to fatigue are also influenced by temperature. Electric-field 
sensitive cycling behavior has been previously reported in yttria-doped $\mathrm{HfO}_{2}^{23}$, and is attributed to the maximization of domain wall density present for pinning when applying fatigue pulses close the $E_{c}$ of the material; at higher fields, domains are larger and fewer domains are present, which suppresses pinning-based fatigue by accumulated charged defects ${ }^{23,31}$. From Figs. 5b. and 5c, some polarization loss is observed when cycling at low voltages but is quite small $\left(<5 \%\right.$ loss of $2 \mathrm{P}_{\mathrm{r}}$ over $10^{7}$ cycles). However, the strongest endurance performance is achieved at $E_{\text {app }}=10.71 \mathrm{MV} / \mathrm{cm}$. Here, the field is large enough to initiate de-pinning of static domains locked in their initial configurations, causing wake-up. The absence of wake-up behavior in the device at lower fields may be related to the decreased mobility of charged defects partially responsible for wake-up, whose thermal and electric energy starvation at low applied bias and temperature suppresses depinning of domain walls.

We note that although oxygen vacancies and trap charges are expected to play significant role in determining the wake-up effects, there is a possibility of additional influence of temperature-dependent strain (due to the difference in thermal expansion coefficients of HZO and LSMO) ${ }^{32-35}$ on the temperature-dependent behavior reported here. Strain calculations for HZO and LSMO are included in Section S5 of the Supplementary Material and reveal that the HZO layer may experience an increase in compressive strain upon cooling from room temperature to $33 \mathrm{~K}$ (i.e. from $-2.65 \%$ to $-4.44 \%$ ). This increase in compressive strain may further reduce the mobility of oxygen vacancies, due to reported influences of such strain on the migration energy for oxygen vacancies in fluorite-based oxide materials ${ }^{42,43}$.In addition, such thermally-induced strain can also directly affect polarization rotation and wake-up effects through complicated electromechanical coupling.

In conclusion, our study demonstrates that PLD-grown $\mathrm{Hf}_{0.5} \mathrm{Zr}_{0.5} \mathrm{O}_{2}$ films exhibit a reduction in apparent polarization and coercive bias as temperature decreases from near room temperature to $33 \mathrm{~K}$ when studied under electric fields below those required for saturation at all temperatures. The magnitude of the wake-up effect is similarly affected, with the greatest increase in polarization after cycling exhibited at room temperature, and the lowest at $33 \mathrm{~K}$. Additionally, under a 5.36 MV/cm field, the material's longevity under cyclic electrical loading increases at low temperature, with characteristics of fatigue manifesting millions of cycles later than when studied near room temperature. Increasing the applied field to $10.71 \mathrm{MV} / \mathrm{cm}$ at $33 \mathrm{~K}$ results in a significant increase in coercive bias and switchable polarization but does not appear to cause saturation. Application of a larger field at $33 \mathrm{~K}$ has little effect on the fatigue behavior but facilitates more rapid wake-up. The apparent reduction in ferroelectric properties with temperature is explained by the increase in HZO's theoretical coercive bias with decreasing temperature, which results in incomplete switching under the $5.36 \mathrm{MV} / \mathrm{cm}$ field in our experiments. It is possible that the temperature-dependent endurance of $\mathrm{HZO}$ is due to the deficiency in thermal energy at cryogenic temperatures, which suppresses the mobility of defects suspected to be oxygen vacancies during wake-up and trapped injected charges during fatigue. Further transport measurements are required to isolate the exact mechanism. Wake-up and fatigue are accelerated at high temperatures where thermal energy is abundant but delayed at cryogenic temperatures where thermal energy is scarce. Increases in compressive strain may additionally further suppress wake-up by way of reducing the mobility of oxygen vacancies, and further analysis of wake-up behavior at lower temperatures may 
provide a greater understanding of its mechanism. These findings highlight the temperaturesensitive ferroelectric switching in epitaxial HZO prepared via PLD and provide insight into the behavior of $\mathrm{HfO}_{2}$-based ferroelectrics across previously understudied range of operating temperatures.

\section{Supplementary Material}

See supplementary material for capacitive hysteresis, an additional comparison of the behavior of the switchable polarization with decreasing temperature that fixes the ratio of the applied field to the measured coercive field, details regarding the determination of the activation energy of the thermally-driven fatigue process, temperature-dependent leakage current measurements, and considerations for the influence of thermally-induced strain on the temperature-dependent ferroelectric behavior of $\mathrm{Hf}_{0.5} \mathrm{Zr}_{0.5} \mathrm{O}_{2}$.

\section{Acknowledgements}

Work at Argonne (J. W. Adkins, S. R. Bakaul, electronic transport experiments, data analysis and contribution to manuscript writing) was supported by the US Department of Energy, Office of Science, Basic Energy Sciences, Materials Sciences and Engineering Division. Use of the Center for Nanoscale Materials was supported by the US Department of Energy, Office of Science, Office of Basic Energy Sciences, under contract no. DE-AC02-06CH11357. J. T. Abiade acknowledges financial support from the U. S. National Science Foundation under grant number NSF-DMR1508220. Financial support from the Spanish Ministerio de Ciencia e Innovación, through the "Severo Ochoa" Programme for Centres of Excellence in R\&D (SEV-2015-0496) and the MAT2017-85232-R (AEI/FEDER, EU), and MAT2015-73839-JIN projects, and from Generalitat de Catalunya (2017 SGR 1377) is acknowledged. J. W. Adkins acknowledges University of Illinois Chicago Pipeline to an Inclusive Faculty (PIF) Program. I. Fina acknowledges Ramón y Cajal contract RYC-2017-22531.

\section{Data A vailability Statement}

The data that support the findings of this study are available from the corresponding author upon reasonable request. 
Figure captions

Fig. 1. Coupled XRD scan of $\mathrm{Hf}_{0.5} \mathrm{Zr}_{0.5} \mathrm{O}_{2} / \mathrm{Laa}_{0.66} \mathrm{Sr}_{0.33} \mathrm{MnO}_{3} / \mathrm{LaNiO}_{3} / \mathrm{CeO}_{2} / \mathrm{YSZ}$ thin films on $\mathrm{Si}$ (100) substrates.

Fig. 2. P-E hysteresis and corresponding I-E behavior for pristine ferroelectric $\mathrm{Hf}_{0.5} \mathrm{Zr}_{0.5} \mathrm{O}_{2}$. Data were collected using a max bias of $4 \mathrm{~V}(7.14 \mathrm{MV} / \mathrm{cm})$ and a hysteresis period of $1 \mathrm{kHz}$.

Fig. 3. (a) P-E hysteresis of pristine $\mathrm{Hf}_{0.5} \mathrm{Zr}_{0.5} \mathrm{O}_{2}$. from $280 \mathrm{~K}$ to $33 \mathrm{~K}$. (b) PUND evolution of switchable polarization up to $7.14 \mathrm{MV} / \mathrm{cm}$ from $280 \mathrm{~K}$ to $33 \mathrm{~K}$. (c) P-E hysteresis of PUND-cycled $\mathrm{Hf}_{0.5} \mathrm{Zr}_{0.5} \mathrm{O}_{2}$. from $280 \mathrm{~K}$ to $33 \mathrm{~K}$. (d) Switchable polarization $\left(\mathrm{P}_{\mathrm{sw}}\right)$ and coercive field $\left(\mathrm{E}_{\mathrm{c}}\right)$ from $280 \mathrm{~K}$ to $33 \mathrm{~K}$ before and after PUND measurements.

Fig. 4. (a) Evolution of memory window of $\mathrm{Hf}_{0.5} \mathrm{Zr}_{0.5} \mathrm{O}_{2}$ with fatigue cycles from $293 \mathrm{~K}$ to $33 \mathrm{~K}$. Inset: Plot of the temperature-dependent function $\operatorname{lnR}(\mathrm{T})$ vs. 1000/T for $\mathrm{T}=293,250 \mathrm{~K}, 220 \mathrm{~K}$, $160 \mathrm{~K}$, and $100 \mathrm{~K}$. (b) Memory window vs. temperature from $\mathrm{T}=293 \mathrm{~K}$ to $33 \mathrm{~K}$ at select cycling milestones.

Fig. 5. (a) P-E hysteresis of pristine $\mathrm{Hf}_{0.5} \mathrm{Zr}_{0.5} \mathrm{O}_{2}$. at $33 \mathrm{~K}$ and $E_{\text {app }}=5.36-10.71 \mathrm{MV} / \mathrm{cm}$. (b) Normalized memory window vs. fatigue cycles at $E_{\text {app }}=5.36-10.71 \mathrm{MV} / \mathrm{cm}$. (c) Plot of normalized memory window vs $E_{\text {app }}$ for pristine $\mathrm{Hf}_{0.5} \mathrm{Zr}_{0.5} \mathrm{O}_{2}$ (black circles) and after $10^{7}$ cycles (red squares). 
Fig. 1

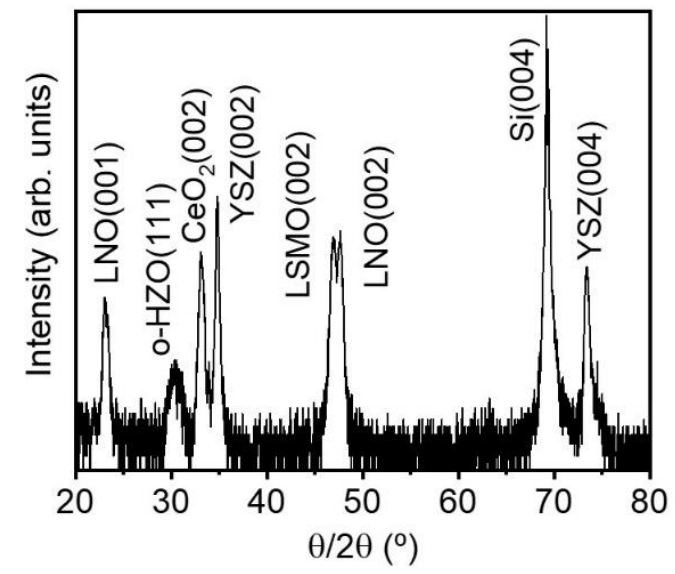


Fig. 2

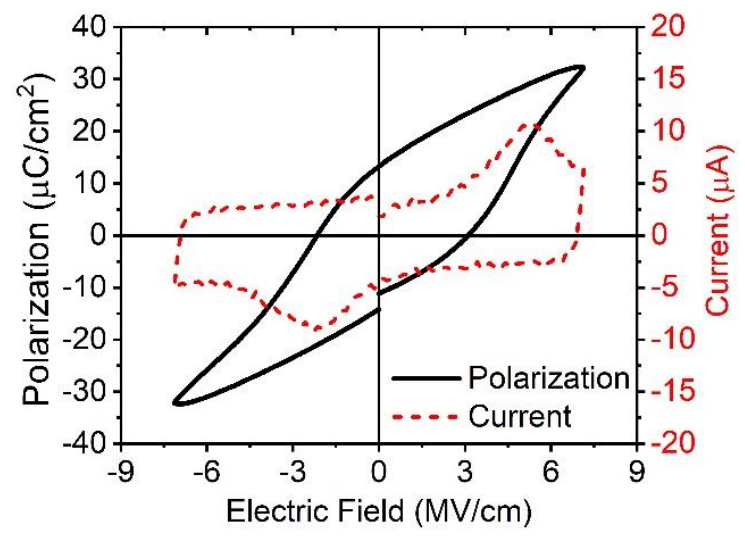


Fig. 3
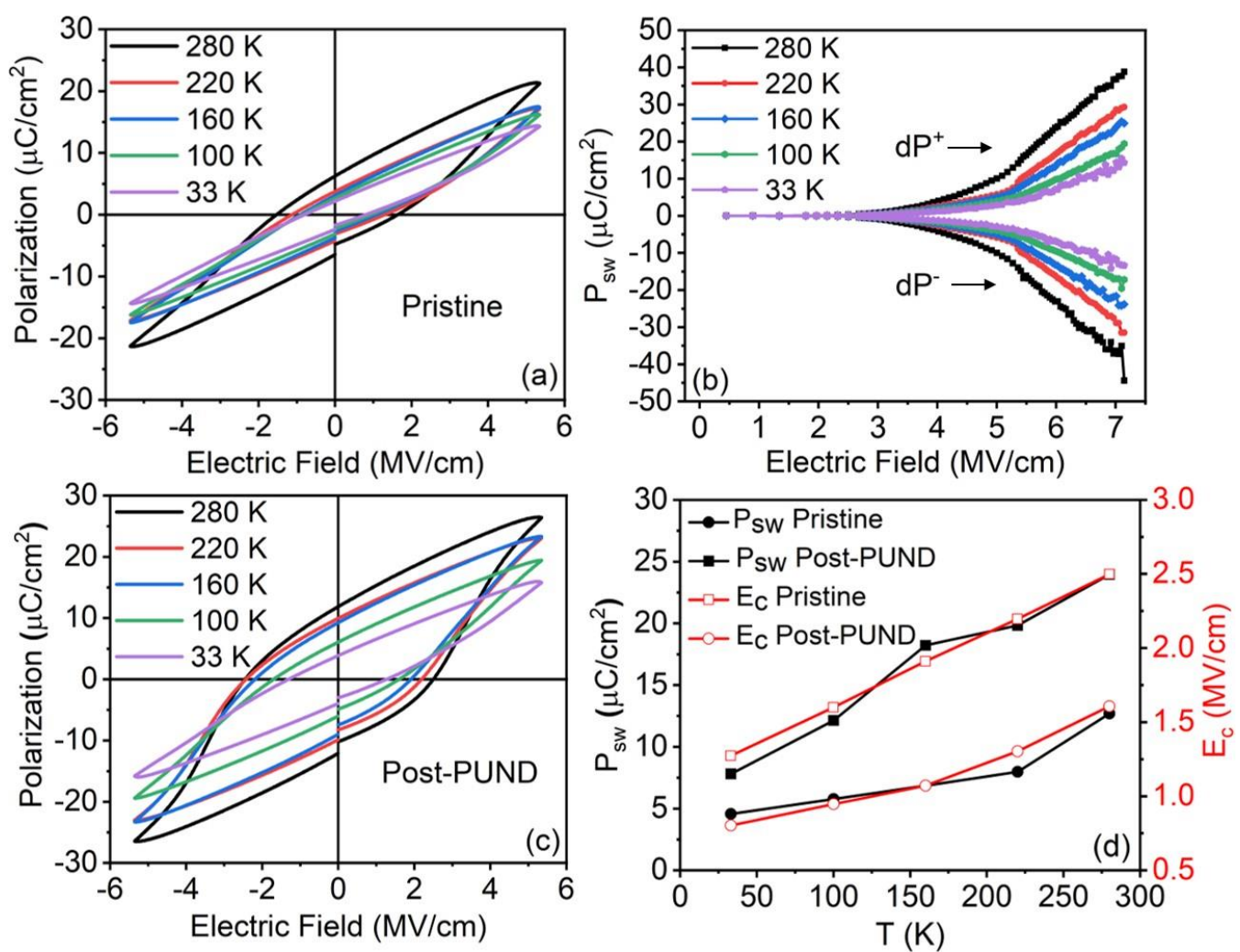
Fig. 4
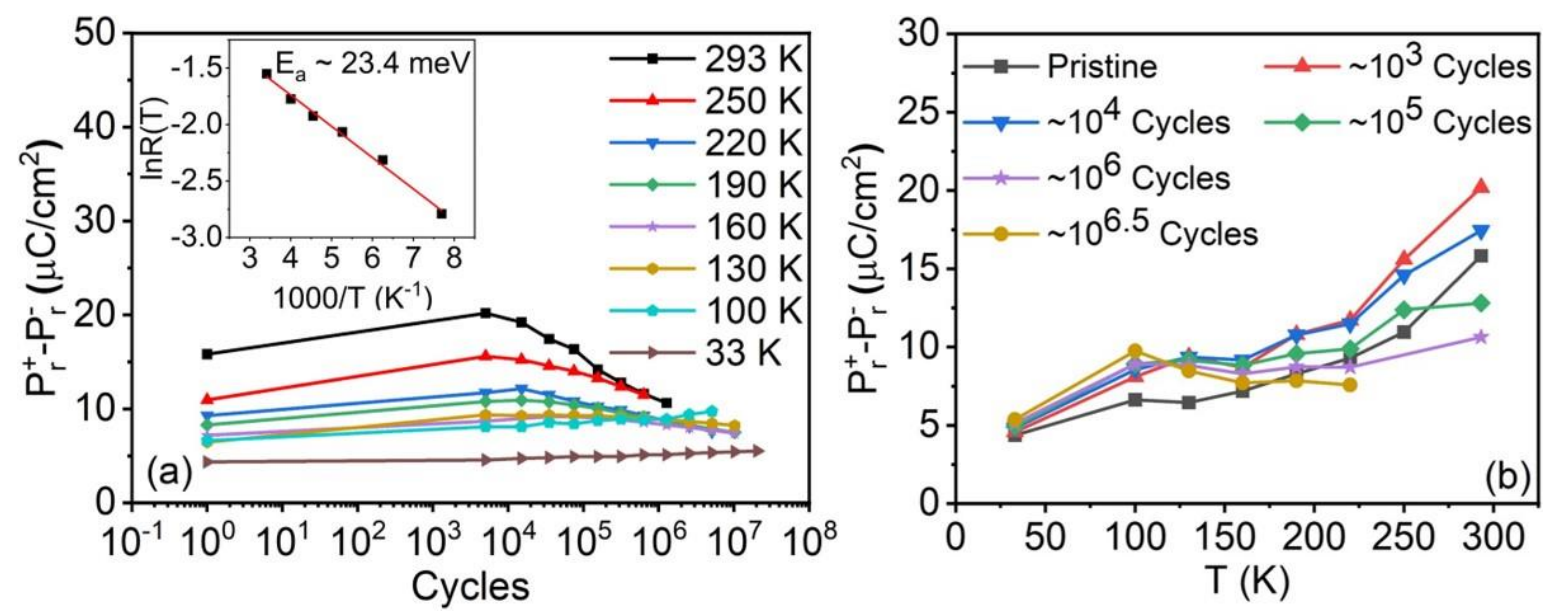
Fig. 5
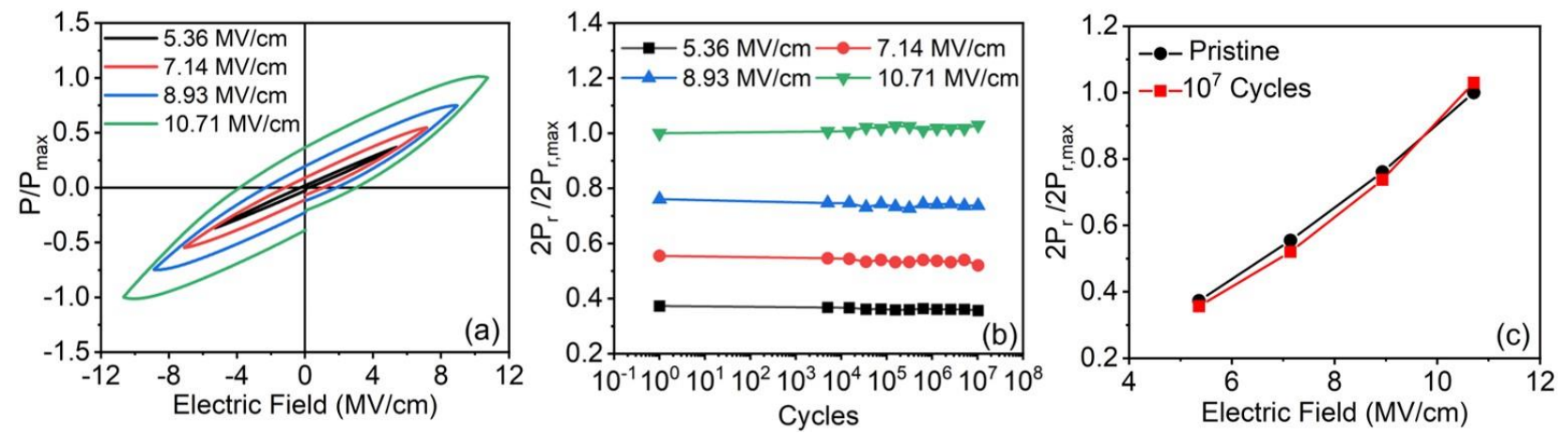


\section{References}

${ }^{1}$ T.S. Böscke, J. Müller, D. Bräuhaus, U. Schröder, and U. Böttger, Appl. Phys. Lett. 99, (2011).

${ }^{2}$ M. Hoffmann, U. Schroeder, C. Künneth, A. Kersch, S. Starschich, U. Böttger, and T. Mikolajick, Nano Energy 18, 154 (2015).

${ }^{3}$ M.H. Park, H.J. Kim, Y.J. Kim, T. Moon, K. Do Kim, Y.H. Lee, S.D. Hyun, and C.S. Hwang, Adv. Mater. 28, 7956 (2016).

${ }^{4}$ J. Müller, T.S. Böscke, D. Bräuhaus, U. Schröder, U. Böttger, J. Sundqvist, P. Kcher, T.

Mikolajick, and L. Frey, Appl. Phys. Lett. 99, 0 (2011).

${ }^{5}$ Z. Fan, J. Chen, and J. Wang, J. Adv. Dielectr. 6, 1 (2016).

${ }^{6}$ S. Mueller, J. Mueller, A. Singh, S. Riedel, J. Sundqvist, U. Schroeder, and T. Mikolajick, Adv. Funct. Mater. 22, 2412 (2012).

${ }^{7}$ S. Mueller, C. Adelmann, A. Singh, S. Van Elshocht, U. Schroeder, and T. Mikolajick, ECS J. Solid State Sci. Technol. 1, N123 (2012).

${ }^{8}$ T. Schenk, S. Mueller, U. Schroeder, R. Materlik, A. Kersch, M. Popovici, C. Adelmann, S. Van Elshocht, and T. Mikolajick, Eur. Solid-State Device Res. Conf. 260 (2013).

${ }^{9}$ T. Shimizu, K. Katayama, T. Kiguchi, A. Akama, T.J. Konno, O. Sakata, and H. Funakubo, Sci. Rep. 6, 1 (2016).

${ }^{10}$ J. Müller, T.S. Böscke, U. Schröder, S. Mueller, D. Bräuhaus, U. Böttger, L. Frey, and T. Mikolajick, Nano Lett. 12, 4318 (2012).

${ }^{11}$ D. Zhou, J. Xu, Q. Li, Y. Guan, F. Cao, X. Dong, J. Müller, T. Schenk, and U. Schröder, Appl. Phys. Lett. 103, (2013).

12 W.L. Warren, B.A. Tuttle, and D. Dimos, Appl. Phys. Lett. 67, 1426 (1995).

${ }^{13}$ C. Brennan, Ferroelectrics 150, 199 (1993).

14 T. Schenk, U. Schroeder, M. Pešić, M. Popovici, Y. V. Pershin, and T. Mikolajick, ACS Appl. Mater. Interfaces 6, 19744 (2014).

${ }^{15}$ M. Pešić, F.P.G. Fengler, L. Larcher, A. Padovani, T. Schenk, E.D. Grimley, X. Sang, J.M. LeBeau, S. Slesazeck, U. Schroeder, and T. Mikolajick, Adv. Funct. Mater. 26, 4601 (2016).

${ }^{16}$ M.H. Park, T. Schenk, C.M. Fancher, E.D. Grimley, C. Zhou, C. Richter, J.M. Lebeau, J.L. Jones, T. Mikolajick, and U. Schroeder, J. Mater. Chem. C 5, 4677 (2017).

${ }^{17}$ E.D. Grimley, T. Schenk, X. Sang, M. Pešić, U. Schroeder, T. Mikolajick, and J.M. LeBeau, Adv. Electron. Mater. 2, (2016).

${ }^{18}$ A. Chouprik, M. Spiridonov, S. Zarubin, R. Kirtaev, V. Mikheev, Y. Lebedinskii, S. Zakharchenko, and D. Negrov, ACS Appl. Electron. Mater. 1, 275 (2019).

${ }^{19}$ J.M.D. Coey, M. Venkatesan, P. Stamenov, C.B. Fitzgerald, and L.S. Dorneles, Phys. Rev. B - 
Condens. Matter Mater. Phys. 72, 3 (2005).

${ }^{20}$ E. Hildebrandt, J. Kurian, M.M. Mller, T. Schroeder, H.J. Kleebe, and L. Alff, Appl. Phys. Lett. 99, (2011).

${ }^{21}$ M.H. Park, C.C. Chung, T. Schenk, C. Richter, M. Hoffmann, S. Wirth, J.L. Jones, T.

Mikolajick, and U. Schroeder, Adv. Electron. Mater. 4, 1 (2018).

${ }^{22}$ S. Starschich, S. Menzel, and U. Böttger, Appl. Phys. Lett. 108, (2016).

${ }^{23}$ D. Zhou, Y. Guan, M.M. Vopson, J. Xu, H. Liang, F. Cao, X. Dong, J. Mueller, T. Schenk, and U. Schroeder, Acta Mater. 99, 240 (2015).

${ }^{24}$ D. Wang, J. Wang, Q. Li, W. He, M. Guo, A. Zhang, Z. Fan, D. Chen, M. Qin, M. Zeng, X. Gao, G. Zhou, X. Lu, and J. Liu, Jpn. J. Appl. Phys. 58, 0 (2019).

${ }^{25}$ J. Lyu, I. Fina, R. Bachelet, G. Saint-Girons, S. Estandía, J. Gázquez, J. Fontcuberta, and F. Sánchez, Appl. Phys. Lett. 114, 0 (2019).

${ }^{26}$ J. Lyu, T. Song, I. Fina, and F. Sánchez, Nanoscale 12, 11280 (2020).

${ }^{27}$ A.F. Devonshire, Adv. Phys. 3, 85 (1954).

${ }^{28}$ M. Vopsaroiu, J. Blackburn, M.G. Cain, and P.M. Weaver, Phys. Rev. B - Condens. Matter Mater. Phys. 82, 1 (2010).

${ }^{29}$ M.H. Park, H.J. Kim, Y.J. Kim, Y.H. Lee, T. Moon, K. Do Kim, S.D. Hyun, F. Fengler, U. Schroeder, and C.S. Hwang, ACS Appl. Mater. Interfaces 8, 15466 (2016).

${ }^{30}$ C.J. Brennan, R.D. Parrella, and D.E. Larsen, Ferroelectrics 151, 33 (1994).

${ }^{31}$ F. Huang, X. Chen, X. Liang, J. Qin, Y. Zhang, T. Huang, Z. Wang, B. Peng, P. Zhou, H. Lu, L. Zhang, L. Deng, M. Liu, Q. Liu, H. Tian, and L. Bi, Phys. Chem. Chem. Phys. 19, 3486 (2017).

${ }^{32}$ N. Farag, M. Bobeth, W. Pompe, A.E. Romanov, and J.S. Speck, Phys. Status Solidi Appl. Mater. Sci. 202, 44 (2005).

${ }^{33}$ R.P. Haggerty, P. Sarin, Z.D. Apostolov, P.E. Driemeyer, and W.M. Kriven, J. Am. Ceram. Soc. 97, 2213 (2014).

${ }^{34}$ D. de Ligny and P. Richet, Phys. Rev. B - Condens. Matter Mater. Phys. 53, 3013 (1996).

${ }^{35}$ H. Inaba, Int. J. Thermophys. 21, 249 (2000).

${ }^{36}$ R.A. De Souza, A. Ramadan, and S. Hörner, Energy Environ. Sci. 5, 5445 (2012).

${ }^{37}$ J. Jiang, X. Hu, W. Shen, C. Ni, and J.L. Hertz, Appl. Phys. Lett. 102, (2013). 